\title{
Prioritizing the Best Areas for Treated Wastewater Use Using RCP 8.5
}

\author{
Sang-Mook Jeon ${ }^{1}$, Eun-Sung Chung ${ }^{1^{*}}$, Yeonjoo Kim², Sang-ho Lee ${ }^{3}$ \\ ${ }^{1}$ Department of Civil Engineering, Seoul National University, Seoul, Republic of Korea; ${ }^{2}$ Korea Environment Institute, Seoul, Re- \\ public of Korea; ${ }^{3}$ Department of Civil Engineering, Pukyung National University, Pusan, Republic of Korea. \\ Email: eschung@seoultech.ac.kr
}

Received 2013

\begin{abstract}
The goal of this study is to develop a new framework that prioritizes the best sites for treated wastewater (TWW) use considering climate change impacts. Fuzzy TOPSIS which is a kind of multi-criteria decision making techniques was introduced to reflect the uncertainty of input data and criteria weighting values. Representative concentration pathway 8.5 scenario was included into the hydrologic simulations for the climate change impact to hydrologic regimes using hydrological simulation program-Fortran (HSPF). Furthermore, all year scenarios were considered to determine the rankings, respectively. It can take into consideration the uncertainty of time periods which always exists in all climate change scenarios. This study can be a baseline to start to combine the fuzzy multi-criteria decision making techniques with robust prioritization for climate change adaptation strategies.
\end{abstract}

Keywords: Adaptation Strategy; Climate Change; Fuzzy TOPSIS; Representative Concentration Pathway

\section{Introduction}

It is clear that some degree of climate change during the next century is now inevitable. Therefore, research over the past twenty years has extensively investigated the potential impacts of climate change on regional hydrology and consequent implications for water resource management systems [1]. Despite these efforts, there is no consensus in the literature on appropriate strategies to cope with non-stationary climate, or even the criteria by which to determine the relative merit of alternative adaptation policies [2].

Although the previous studies showed robust decision making (RDM) technique for climate change adaptation ([2-4]), this study will propose two major improvements. First, Fuzzy concept can be added to RDM since the future climate values and their impact analysis results show the large variances if various scenarios and long periods ( 2100) are considered. Second, some multi-criteria decision making (MCDM) techniques can be added to RDM for the consideration of various criteria, since different MCDM methods use different decision philosophies.

Therefore, this study will describe a new analytic framework for identifying the priorities of climate change adaptation strategies that may help resolve the above uncertainty. Fuzzy concept will be used to reduce the un-

\footnotetext{
${ }^{*}$ Corresponding author.
}

certainty in both weights of evaluation criteria and the crisp input data from hydrologic analyses and TOPSIS was applied to consider the various evaluation results.

\section{Methodology}

\subsection{Procedure}

The traditional framework for assessing climate change adaptation strategies rests on the assumption that we can predict the future. Therefore, this prediction-based policy analysis requires the exact answer to the question "what is likely to happen in the future?”. It, however, is wellknown fact that the question "what actions should we take, given that we cannot predict the future?" becomes more important [2].

In this framework, a new framework different from the many previous RDM research will be proposed to consider two kinds of uncertainty. First, Fuzzy concept is added to RDM since the future climate values and their impact analysis results show the large variances if various scenarios and long periods ( 2100) are considered. Each period (or year) can be assumed to be an individual scenario. Second, an MCDM technique can be added to $\mathrm{RDM}$ in order to consider anthropogenic criteria as well as engineering indicators.

To include these points, this study consists of four steps: 1 ) to derive various adaptation strategies (or feasi- 
ble locations for adaptation strategies; (2) to establish the evaluation criteria including environmental factors as well as anthropogenic issues (social and economic); 3) to derive the decision matrix using data collections and the hydrological analyses from hydrological simulation program-fortran (HSPF) model; and (4) to rank all adaptation strategies using Fuzzy multi-criteria approach.

\subsection{Overview of RCP}

The representative concentration pathways (RCPs) were developed by four integrated assessment modeling teams (8.5, 6.5, 4.5 and 2.5) around the world and represent four potential different global emissions pathways that would lead to the widest possible range of radiative forcing by the end of this century [5]. More information about the RCPs and official data from the scenarios is available from IIASA. [5] summarizes global $\mathrm{CO}_{2}$ emissions and the resulting $\mathrm{CO}_{2}$ concentrations across the GCAM's representation of the four RCPs provide an overview of the GCAM model. For the simple application, this study used RCP8.5 which assumed the highest CO2 emissions per year. RCP8.5W/ $\mathrm{m}^{2}$ developed by the IIASA/MESSAGE modeling team in Austria [6]. This corresponds to a world where greenhouse gas emissions continue to rise resulting in atmospheric $\mathrm{CO}_{2}$ concentration that exceeds 900 ppmv by 2100 .

\subsection{Fuzzy TOPSIS}

The TOPSIS method was developed to solve MCDM problem in which preference information is not articulated [7]. The technique is based on the concept that the ideal alternative has the best values for all attributes, whereas the negative ideal is the alternative with all of the worst attribute values. A TOPSIS solution is defined as the alternative that is simultaneously farthest from the negative ideal and closest to the ideal alternative. According to [8], TOPSIS has four advantages: (1) a sound logic that represents rational human choices; (2) a scalar value that simultaneously accounts for the best and worst alternatives simultaneously; (3) a simple computation process that can be easily programmed; and (4) the performance measures for attributes can be visualized for all alternatives on a polyhedron for any two dimensions. Here the detailed procedure for the Fuzzy TOPSIS is presented as the procedure for the TOPSIS is the almost same except for using no FTNs.

[9] proposed a multi-objective fuzzy pattern recognition model to provide a global evaluation for every district with respect to all criteria. According to the maximum principle of membership degree, one can select the desired alternative from $n$ available districts. They defined the optimum membership degree of each district as:

$$
\mu_{j}=\frac{\left(d_{j}^{-}\right)^{2}}{\left(d_{j}^{*}\right)^{2}+\left(d_{j}^{-}\right)^{2}}
$$

where $d_{j}^{*}$ and $d_{j}^{-}$are the synthetically weighted distance of district $j$ to the best and worst districts, respecttively.

To calculate the distances of district $A_{j}$ to the ideal (best) and anti-ideal (worst) districts, the fuzzy ideal weight distance is defined as follows:

$$
\begin{aligned}
& d_{j}^{*}=\left[\sum_{i=1}^{m}\left(d\left(\tilde{r}_{i j}^{\tilde{w}}, \tilde{r}_{i}^{*} \tilde{w}\right)\right)^{q}\right]^{1 / q} \\
& d_{j}^{-}=\left[\sum_{i=1}^{m}\left(d\left(\tilde{r}_{i j}^{\tilde{w}}, \tilde{r}_{i}^{-\tilde{w}}\right)\right)^{q}\right]^{1 / q}
\end{aligned}
$$

where $\tilde{r}_{i j}^{w}$ is the weighted performance value of district $A_{j}$ with respect to criterion $C_{i}$, and $m$ is the number of criteria.

\section{Results and Discussions}

\subsection{Feasible Locations for TWW Use}

Given the necessity of TWW use and its rapidly growing significance, it has become necessary to establish a methodology for locating suitable sites for TWW use. However, no site selection problems have been applied to TWW use for instream flow. However, there is still a huge gap between the quantity of sewage generated and the available treatment capacity, and a decision support system is urgently needed to select appropriate locations and best-fit quantities within the supply [10]. TWW use has a critical disadvantage in that it leads to water quality deterioration. Highly treated wastewater can be used instead, but requires a tremendous budget and most countries cannot satisfy residents' demand with such water. Thus, the trade-off between flow regime enrichment and water quality aggravation must be determined with caution.

We derived ten suitable sites, or alternatives, for TWW use in the Anyangcheon watershed by considering the streamflow quantity during the dry period and each sub-watershed area (Figure 1). To determine the appropriate quantity of TWW use, each sub-watershed's area was used. TWW quantity to maintain the environmental instreamflow requirement is closely related to the demand and supply of the study area. In this step, the reference quantity, 22,000 $\mathrm{m}^{3} /$ day in HU was used since it is already under operation. Therefore, the other quantities were calculated in proportion to the area of HU. The selected quantities for all alternatives are listed in Table $\mathbf{1 .}$ Also, BOD conc. for TWW use with a target BOD of 4.7 $\mathrm{mg} / \mathrm{L}$ was determined since it is the highly-treated effluxent standard of the Anyang wastewater treatment for in- 
stream flow.

\subsection{Establishing Criteria}

Based on the concept of the sustainable development, all criteria used to quantify the model's effectiveness were determined by expert researchers and local governmental officials because the process required discussion and refinement. The structure of the selected criteria is shown in Table 2. Population and population density were chosen as influencing both water quantity and quality because these criteria lead to relevant environmental pressures. Urban area ratio, streamflow seepage, watershed slope, and groundwater withdrawal were the pressures assumed for water quantity, while untreated wastewater intrusion, and ratio of covered stream interval were the pressures assumed for water quality. These pressures affect the ratio of low flow to hydrological instream flow for water quantity and the ratio of BOD ave. conc. to the target concentration for water quality. This study selected transformation ratios based on low flow and BOD conc. due to adaptation strategy because responses to these indicators can lead to remedial measures and planning actions.

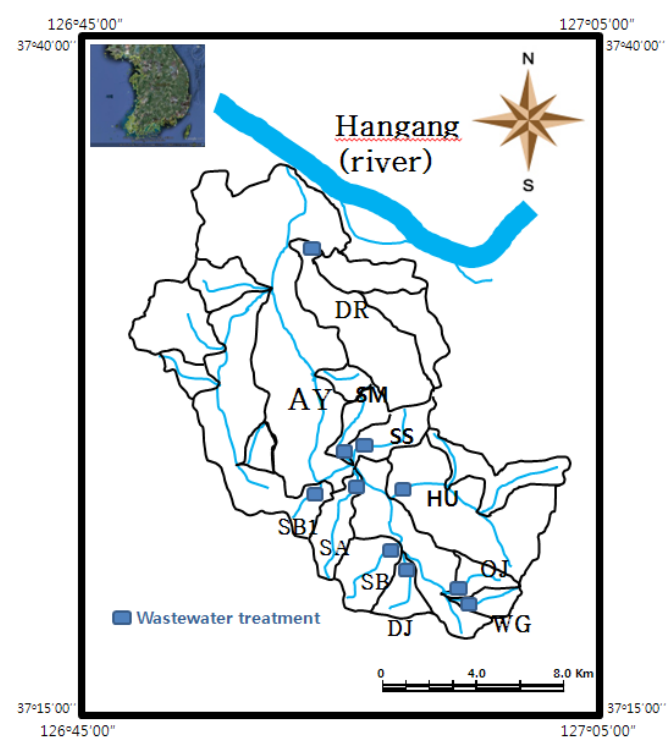

Fig. 1. Map of study area.

Table 1. List of TWW use quantity for each sub-watershed.

\begin{tabular}{cccccc}
\hline tlA & $\begin{array}{c}\text { aerA } \\
\left(\mathrm{km}^{2}\right)\end{array}$ & $\begin{array}{c}\text { ytitnauQ } \\
\left(\mathrm{m}^{3} / \mathrm{d}\right)\end{array}$ & tlA & $\begin{array}{c}\text { aerA } \\
\left(\mathrm{km}^{2}\right)\end{array}$ & $\begin{array}{c}\text { ytitnauQ } \\
\left(\mathrm{m}^{3} / \mathrm{d}\right)\end{array}$ \\
\hline GW & 3.78 & 2000 & AS & 8.07 & 4000 \\
JO & 4.26 & 3000 & MS & 5.39 & 3000 \\
JD & 5.33 & 3000 & SS & 13.18 & 7000 \\
BS & 10.30 & 5000 & 1BS & 4.59 & 3000 \\
UH & 44.55 & 21000 & RD & 41.61 & 20000 \\
\hline
\end{tabular}

Table 2. Weighting values of eleven evaluation criteria.

\begin{tabular}{lc}
\hline Criteria & Weights \\
\hline Population & 0.03 \\
Population density & 0.13 \\
Urban area ratio & 0.04 \\
Streamflow seepage & 0.02 \\
Groundwater withdrawal & 0.03 \\
Untreated wastewater intrusion & 0.04 \\
Ratio of covered stream interval & 0.03 \\
Ratio of low flow to hydrologic instreamflow & 0.09 \\
Ratio of increased low flow to hydrological instreamflow & 0.22 \\
Ratio of BOD average concentration to target quality & 0.10 \\
Ratio of decreased BOD average concentration to target & 0.25 \\
quality & \\
\hline
\end{tabular}

All criteria had their internal impacts reclassified to a common scale, making it necessary to determine each criterion's relative impact in prioritizing best sites for TWW use. Weights were assigned to the criteria to indicate their relative importance, and different weights directly influenced the DM results for best sites selection with TWW use. Consequently, it was necessary to determine the rationality and veracity of the criteria weights. We used two methods to determine the weights of all the criteria, individual interviews with 31 experts and the fuzzy concept. The respondent group consisted of local governmental officials and researchers working in river management, and the TFNs of the weighting values were derived using the survey results from the 31 respondents.

\subsection{Evaluation of Alternatives}

Using HSPF model which has already verified in [11], ten simulated results for water quantity and quality were averaged and the results with and without each alternative were compared. To quantify the effectiveness, the performance measure "effectiveness" was used ([4,1113]). In this study, it was applied to low flow and BOD concentrations. They can be calculated using the following equation:

$$
f(i)=\frac{x^{\prime}(i)-x(i)}{x(i)}
$$

where $f$ is effectiveness of alternative $i, x(i)$ is the original value without adaptation strategy and $x^{\prime}(i)$ is the transformed value.

The calculated effectiveness is shown in Table 3. As expected, the criteria related to water quantity improved, and low flows increased in all cases. However, the criteria related to water quality (i.e., BOD conc.) worsened even with TWW use (BOD conc. of $4.7 \mathrm{mg} / \mathrm{L}$ ). TWW BOD conc. was much higher than those in natural stream flows, indicating TWW use had harmful effects on water quality in the urban watershed. In addition, In addition, comparing with the results of historical climate data, the positive effectiveness to low flow increases except WG if 
climate change happens while the negative decreases. That is, complicate mixing effects can happen when climate change impact is considered for water resources planning and management. Therefore, given the positive and negative effects of TWW use on the urban watershed water cycle, a best location selection must be approached with caution when TWW uses are considered due to the risk of compromised quantity.

Furthermore, the effectiveness of the alternatives to water quantity and quality varied significantly by year. Therefore, the low flows with adaptation strategies are derived as shown in Table 4 using 90-year climate data (2010-2099) from RCP8.5. As a result, the effectiveness of TWW use varied every year dramatically because the predicted climate conditions from selected general circulation models and climate change scenarios varies unexpectedly. This inter-annual variability in the simulated criteria suggested that the average data over multiple years could not adequately represent the simulation results, necessitating techniques that would better represent such variability. Therefore, the TFN concept can be adopted to derive the performance values of all the alternatives.

Table 3. Effectiveness ratios to water quantity and quality by three time scenarios and historical series.

\begin{tabular}{|c|c|c|c|c|}
\hline woL Flow & 2010-2039 & 2040-2069 & 2070-2099 & Historical \\
\hline WG & $259 \%$ & $250 \%$ & $258 \%$ & $289 \%$ \\
\hline OJ & $68 \%$ & $60 \%$ & $67 \%$ & $51 \%$ \\
\hline DJ & $74 \%$ & $64 \%$ & $74 \%$ & $66 \%$ \\
\hline SB & $62 \%$ & $52 \%$ & $61 \%$ & $49 \%$ \\
\hline HU & $55 \%$ & $48 \%$ & $55 \%$ & $44 \%$ \\
\hline SA & $54 \%$ & $46 \%$ & $54 \%$ & $42 \%$ \\
\hline $\mathrm{SM}$ & $39 \%$ & $32 \%$ & $38 \%$ & $31 \%$ \\
\hline SS & $125 \%$ & $114 \%$ & $125 \%$ & $93 \%$ \\
\hline SB1 & $131 \%$ & $124 \%$ & $137 \%$ & $105 \%$ \\
\hline DR & $90 \%$ & $80 \%$ & $90 \%$ & $71 \%$ \\
\hline BOD Conc. & 2010-2039 & 2040-2069 & 2070-2099 & Historical \\
\hline WG & $-23 \%$ & $-21 \%$ & $-23 \%$ & $-30 \%$ \\
\hline OJ & $-79 \%$ & $-75 \%$ & $-73 \%$ & $-53 \%$ \\
\hline DJ & $-42 \%$ & $-39 \%$ & $-38 \%$ & $-31 \%$ \\
\hline SB & $-52 \%$ & $-48 \%$ & $-47 \%$ & $-35 \%$ \\
\hline $\mathrm{HU}$ & $-48 \%$ & $-40 \%$ & $-45 \%$ & $-33 \%$ \\
\hline SA & $-72 \%$ & $-67 \%$ & $-65 \%$ & $-49 \%$ \\
\hline SM & $-96 \%$ & $-91 \%$ & $-89 \%$ & $-65 \%$ \\
\hline SS & $-148 \%$ & $-136 \%$ & $-134 \%$ & $-98 \%$ \\
\hline SB1 & $-58 \%$ & $-69 \%$ & $-60 \%$ & $-41 \%$ \\
\hline DR & $-64 \%$ & $-61 \%$ & $-60 \%$ & $-44 \%$ \\
\hline
\end{tabular}

\subsection{Fuzzy Multi-criteria Approach}

This study used an MCDM technique to derive a ranking of all feasible alternatives. For the sustainability, it is a general fact that various criteria should be considered. Thus, this study compared the traditional decision making results with those of TOPSIS as shown in Figure 2. The traditional method generally includes just engineering criteria such as low flow and BOD conc. while MCDM considers anthropogenic criteria such as population, population density, urban area ratio and so on. As a result, some watersheds such as WG, DJ, SB and SS showed large differences. Therefore, social factors should be include to select the effective adaptation strategies for climate change.

Based on the section 3.3, this study adopted triangular fuzzy number (TFN) to reflect the variations of low flows and BOC conc according to all years of RCP8.5. The performance values of low flow and BOD conc. were normalized to apply Fuzzy TOPSIS. Meanwhile, performance values for basic components were assumed to be fuzzy because their distributions were not derived, and we set the upper $10 \%$ and lower $10 \%$ of the average results as the upper and lower limits of the TFNs.

Table 4. Simulated ave. and key percentile low flows from RCP8.5.

\begin{tabular}{ccccc}
\hline \multirow{2}{*}{ Low } & \multirow{2}{*}{$\begin{array}{c}\text { Ave. } \\
\text { Flow }\end{array}$} & Low flow & \multicolumn{3}{c}{ Percentile values of low flow } \\
\cline { 3 - 5 } & & $10 \%$ & $50 \%$ & $90 \%$ \\
\hline WG & 0.0003 & 0.0000 & 0.0000 & 0.0000 \\
OJ & 0.0849 & 0.0991 & 0.0850 & 0.0680 \\
DJ & 0.0834 & 0.0991 & 0.0821 & 0.0651 \\
SB & 0.1551 & 0.1841 & 0.1543 & 0.1218 \\
HU & 0.6935 & 0.8297 & 0.6966 & 0.5465 \\
SA & 0.1355 & 0.1642 & 0.1359 & 0.1048 \\
SM & 0.1215 & 0.1472 & 0.1218 & 0.0934 \\
SS & 0.1466 & 0.1642 & 0.1472 & 0.1246 \\
SB1 & 0.0608 & 0.0736 & 0.0595 & 0.0510 \\
DR & 0.4918 & 0.5692 & 0.4927 & 0.4021 \\
\hline
\end{tabular}

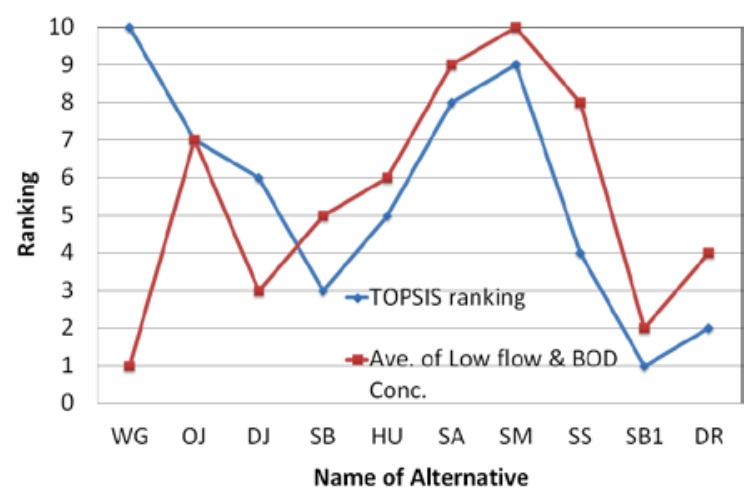

Figure 2. Comparison of rankings considering between various and engineering criteria. 
The fuzzy ideal weight distances of the alternative $A_{j}$ values to the ideal (best) and anti-ideal (worst) points were calculated using Eqs. 2 and 3. The separation between the FPIS and FNIS. These results and Eq. 1 were used to calculate the optimum membership degree of each alternative to water quantity and quality, respectively. Ave. rankings of every 10-year result are listed in Figure 3. As a result, four alternatives such as OJ, SM, $\mathrm{HU}$, and DJ showed different rankings according to the time scenarios. That is, to derive water resources plan, various time scenarios and climate change model should be considered for the robust prioritization.

To check the efficiency of fuzzy concept, TOPSIS was applied to the same procedure. The results are shown in Figure 4. Comparing with Figure 3, the ranking variations are more severe. It can be concluded that Fuzzy TOPSIS show more robust ranking than TOPSIS since it consider the uncertainty of all data.

\section{Conclusions}

This study developed a multi-criteria approach with fuzzy set theory to the problem of location selection for TWW use in a Korean urban watershed. Fuzzy TOPSIS was introduced to prioritize the best sites. Criteria involving social and environmental components were also

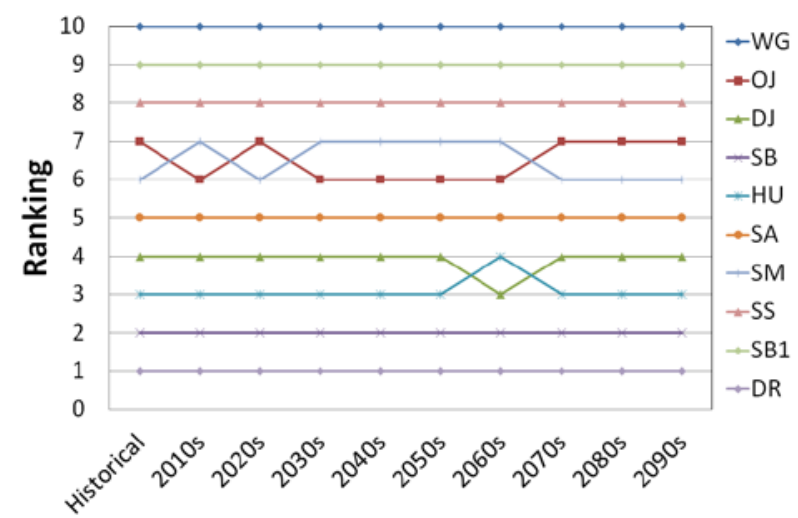

Figure 3. Ranking time series using Fuzzy TOPSIS.

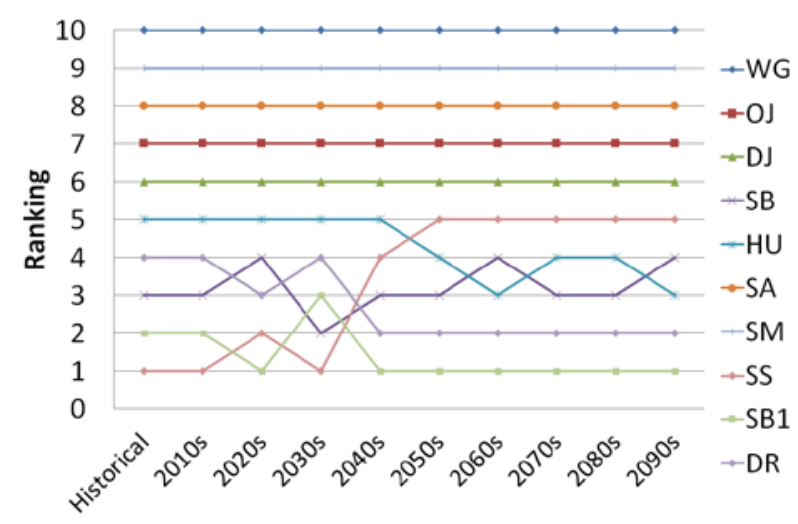

Figure 4. Ranking time series using TOPSIS. selected. The following conclusions were made based on the results of the study.

1) MCDM method can be used to include various evaluation criteria for sustainable development. Criteria were considered based on an integrated framework including social and environmental components, and their weighting values were objectively derived using individual interviews.

2) Fuzzy TOPSIS can be used to derive the robust prioritization of adaptation strategies for climate change since it can reflect the uncertainty of input data and criteria weighting values.

This study can be a baseline to start to combine the fuzzy MCDM techniques with robust prioritization for climate change adaptation strategies

\section{Acknowledgements}

This research was supported by a grant (11-TI-C06) from Construction Technology Innovation Program funded by Ministry of Land, Transport and Maritime Affairs of Korean government.

\section{REFERENCES}

[1] B. Nijssen, G.M. O’Donnell, A.F. Hamlet and D.P. Lettenmaier, "Hydrologic sensitivity of global rivers to climate change,” Climatic Change, Vol. 50, No. 1, 2001, pp. 143-175.

[2] R.J. Lempert, M.E. Schlesinger, "Robust strategies for abating climate change,” Climatic Change, Vol. 45, No. 3, 2000, pp. 387-401.

[3] S. Dessai, X. Lu, J.S. Risbey, "On the role of climate scenarios for adaptation planning.” Global Environmental Change, Vol. 15, 2005, pp. 87-97.

[4] J.S. Yang, E.S. Chung, S.U. Kim, T.W. Kim, "Prioritization of water management under climate change and urbanization using multi-criteria decision making methods.” Hydrology and Earth System Sciences, Vol. 16, 2012, pp. 801-814.

[5] J.J. Dooley, and K.V. Calvin, "Temporal and spatial deployment of Carbon Dioxide capture and storage technologies across the Representative Concentration Pathways." Energy Procedia, Vol. 4, 2011, pp. 58455852.

[6] K. Riahi, A. Gruebler, N. Nakicenovic, "Scenarios of long-term socio-economic and environmental development under climate stabilization”, Technological Forecasting and Social Change, Vol. 74, 2007, pp. 887-935.

[7] C.L. Hwang, K. Yoon, "Multiple Attributes Decision Making Methods and Applications,” Springer, Heidelberg, 1981.

[8] H.S. Shih, H.J. Shyur, E.S. Lee, "An extension of TOPSIS for group decision making," Mathematical Computer Modeling, Vol. 45, 2007, pp. 801-813.

[9] H.C. Zhou, G.L. Wang, and Q. Yang, “A multi-objective 
fuzzy pattern recognition model for assessing groundwater vulnerability based on the DRASTIC system," Hydrological Science Journal, Vol. 44, 1999, pp. 611-618.

[10] J. Ganoulis, "Evaluating alternative strategies for wastewater recycling and reuse in the Mediterranean area," Water Science and Technology: Water Supply, Vol. 3, 2003, pp. 11-19.

[11] E.S. Chung, K.S. Lee, "Prioritization of water management for sustainability using hydrologic simulation model and multi-criteria decision making techniques,” Journal of Environmental Management, Vol. 91, 2009, pp. 1502-1511.

[12] E.S. Chung, W.P. Hong, K.S. Lee, S.J. Burian, "Integrated use of a continuous simulation model and multi-attribute decision making for ranking urban watershed management alternatives.” Water Resources management, Vol. 25, pp. 641-658.

[13] Y. Kim, E.S. Chung, "Integrated assessment of climate change and urbanization impact on adaptation strategies: A case study in two small Korean watersheds." Climatic Change, DOI 10.1007/s10584-012-0612-4. 